\title{
Mixed Upward Planarization - Fast and Robust ${ }^{\star}$
}

\author{
Martin Siebenhaller and Michael Kaufmann \\ Universität Tübingen, WSI, Sand 14, 72076 Tübingen, Germany \\ \{siebenha, mk\}@informatik.uni-tuebingen.de
}

\section{Introduction}

In a mixed upward drawing of a graph $G=(V, E)$ all directed edges $E_{D} \subseteq E$ are represented by monotonically increasing curves. Mixed upward drawings arise in applications like UML diagrams where such edges denote a hierarchical structure. The mixed upward planarization is an important subtask for computing such drawings. We outline a fast and simple heuristic approach that provides a good quality and can be applied to larger graphs as before in reasonable time. Unlike other Sugiyama-style [4] approaches, the quality is comparable to the GT based approach [2] even if there are only few directed edges. Furthermore, the new approach is particularly suitable for extensions like clustering and swimlanes.

\section{Planarization Approach}

We assume that the subgraph induced by the directed edges is acyclic. Our heuristic approach consists of the following three steps:

1. Construction of an upward drawing: We use Sugiyama's approach 44 with a special layering strategy to construct an upward drawing of $G$ including all edges. Common layering strategies are mainly optimized to produce short edges and involve a lot of crossings. Our new layering stems from the vertex ordering obtained in the first phase of the GT-heuristic. To calculate the ordering we use a variant that guarantees a monotonically increasing direction of the directed edges and runs in $O\left(|V|^{2}\right)$ 2. The layer of a vertex corresponds to its position in this ordering. The crossing reduction is done by a layer-by-layer sweep. We apply a fast variant that keeps the number of dummy vertices small and runs in time $O((|V|+|E|) \log |E|)$ [3]. For the horizontal coordinate assignment we use a linear time approach based on the linear segments model [1].

2. Construction of an upward planar embedding: The embedding of the upward drawing of $G$ can be constructed by detecting all crossings and replace them by dummy vertices. This can be done with a sweep-line approach in time $O((|E|+c) \log |E|)$, where $c$ denotes the number of crossings.

3. Rerouting of undirected edges: Undirected edges are handled too restrictive because they can be routed non-monotonically. Hence we reroute each undirected edge with at least one crossing in randomized order using shortest

\footnotetext{
* This work has been supported by DFG-grant Ka812/8-2.
} 
path computations in the extended dual graph. The runtime is $O((|V|+c)|E|)$. Thus, the overall runtime of our new approach is $O((|E|+c) \log |E|+(|V|+c)|E|)$.

\section{Experiments}

For our experiments we used connected directed acyclic random graphs with density 2. In the first experiment (Fig. 3(a)) we compared the number of crossings (using the median heuristic) induced by our new layering to the crossing number induced by a longest path layering, the popular simplex layering and a layering based on a topological sorting of the vertices where each vertex is assigned to exactly one layer. The results indicate that our improvement does not depend solely on the sparse layers. In the second experiment we compared our new approach to the GT based approach described in [2] that runs in $O\left(|V||E|^{2}+\right.$ $\left.(|V|+c)^{2}|E|\right)$. We compared random graphs where $1 / 4(2 / 3)$ of the edges were directed. As the results in Fig. 3(b) show, the quality in terms of crossings is competitive to the GT based approach. Concerning the required time we clearly outperform the GT based approach (Fig. 3(c)). All experiments were performed on a Pentium 4 System, $3 \mathrm{GHz}, 1024$ MB RAM and Windows XP.

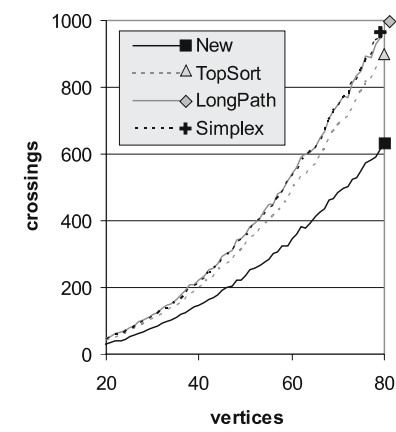

(a)

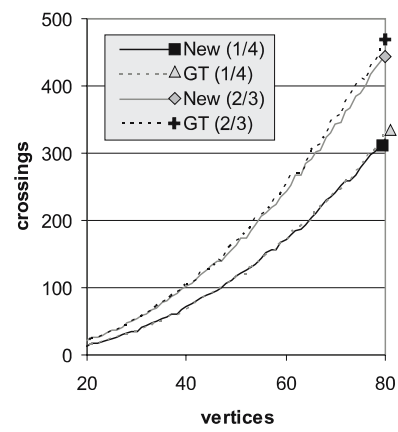

(b)

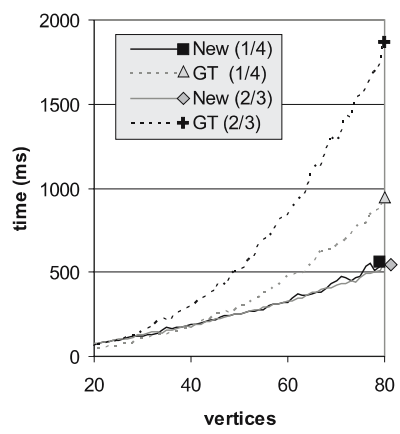

(c)

Fig. 1. Results of our experiments (averaged over 100 passes)

\section{References}

1. U. Brandes and B. Köpf: Fast and Simple Horizontal Coordinate Assignment. Proceedings of Graph Drawing 2001, Springer LNCS 2265, pp. 31-44, 2001.

2. M. Eiglsperger, M. Kaufmann and F. Eppinger: An Approach for Mixed Upward Planarization. J. Graph Algorithms Appl., 7(2): pp. 203-220, 2003.

3. M. Eiglsperger, M. Siebenhaller and M. Kaufmann: An Efficient Implementation of Sugiyama's Algorithm for Layered Graph Drawing. Proceedings of Graph Drawing 2004, Springer LNCS 3383, pp. 155-166, 2005.

4. K. Sugiyama, S. Tagawa and M. Toda: Methods for Visual Understanding of Hierarchical System Structures. IEEE Transactions on Systems, Man and Cybernetics, SMC-11(2): pp. 109-125, 1981. 